\title{
Intraoperative real-time near-infrared optical imaging for the identification of metastatic brain tumors via microscope and exoscope
}

\author{
*Jun Muto, MD, PhD, ${ }^{1}$ Yutaka Mine, MD, PhD, ${ }^{2}$ Yu Nakagawa, MD, PhD, ${ }^{2}$ Masahiro Joko, MD, PhD, ${ }^{1}$ \\ Hiroshi Kagami, MD, ${ }^{2}$ Makoto Inaba, MD, ${ }^{2}$ Mitsuhiro Hasegawa, MD, PhD, ${ }^{1}$ \\ John Y. K. Lee, MD, MSCE, ${ }^{3}$ and Yuichi Hirose, MD, PhD' \\ ${ }^{1}$ Department of Neurosurgery, Fujita Health University, Aichi; ${ }^{2}$ Department of Neurosurgery, Saiseikai Yokohama Tobu Hospital, \\ Kanagawa, Japan; and ${ }^{3}$ Department of Neurosurgery, University of Pennsylvania, Philadelphia, Pennsylvania
}

\begin{abstract}
OBJECTIVE As chemotherapy and radiotherapy have developed, the role of a neurosurgeon in the treatment of metastatic brain tumors is gradually changing. Real-time intraoperative visualization of brain tumors by near-infrared spectroscopy (NIRS) is feasible. The authors aimed to perform real-time intraoperative visualization of the metastatic tumor in brain surgery using second-window indocyanine green (SWIG) with microscope and exoscope systems.

METHODS Ten patients with intraparenchymal brain metastatic tumors were administered $5 \mathrm{mg} / \mathrm{kg}$ indocyanine green (ICG) 1 day before the surgery. In some patients, a microscope was used to help identify the metastases, whereas in the others, an exoscope was used.

RESULTS NIRS with the exoscope and microscope revealed the tumor location from the brain surface and the tumor itself in all 10 patients. The NIR signal could be detected though the normal brain parenchyma up to $20 \mathrm{~mm}$. While the mean signal-to-background ratio (SBR) from the brain surface was $1.82 \pm 1.30$, it was $3.35 \pm 1.76$ from the tumor. The SBR of the tumor $(p=0.030)$ and the ratio of Gd-enhanced T1 tumor signal to normal brain (T1BR) $(p=0.0040)$ were significantly correlated with the tumor diameter. The SBR of the tumor was also correlated with the T1BR $(p=0.0020)$. The tumor was completely removed in 9 of the 10 patients, as confirmed by postoperative Gd-enhanced MRI. This was concomitant with the absence of NIR fluorescence at the end of surgery.
\end{abstract}

CONCLUSIONS SWIG reveals the metastatic tumor location from the brain surface with both the microscope and exoscope systems. The Gd-enhanced T1 tumor signal may predict the NIR signal of the metastatic tumor, thus facilitating tumor resection.

https://thejns.org/doi/abs/10.3171/2020.10.FOCUS20767

KEYWORDS near-infrared; second-window indocyanine green; brain tumor surgery; fluorescence

A PPROXIMATELY $10 \%-26 \%$ of all patients with cancer in Japan develop brain metastases. ${ }^{1}$ In select patients whose neurological status is compromised by mass effect from metastases, performance status can be rapidly improved with resection of the brain lesion. This in turn facilitates the treatment of the primary cancer, which comprises the administration of appropriately selected multidisciplinary treatment that improves the function and quality of life for each patient, thus enhancing long-term survival. Furthermore, excision is the only means that enables tissue diagnosis. The im- portance of genomic medicine and individualization has been reaffirmed in recent years, and they seem promising for improving the accuracy and reliability of resections of metastases to the brain. Real-time intraoperative visualization of brain tumors by indocyanine green (ICG) reportedly facilitates visualization of the tumor dye through the normal brain and dura mater, referred to as the second-window ICG (SWIG). In this technique, $5 \mathrm{mg}$ / $\mathrm{kg}$ ICG is introduced approximately 24 hours before surgery. Several reports have affirmed that SWIG is helpful in the removal of brain tumors..$^{2-4}$

ABBREVIATIONS ICG = indocyanine green; NIR = near-infrared; NIRS = NIR spectroscopy; ROI = region of interest; SBR = signal-to-background ratio; SWIG = secondwindow ICG; T1BR = ratio of Gd-enhanced T1 tumor signal to normal brain; 5-ALA = 5-aminolevulinic acid.

SUBMITTED August 31, 2020. ACCEPTED October 22, 2020.

INCLUDE WHEN CITING DOI: 10.3171/2020.10.FOCUS20767.

* J.M. and Y.M. contributed equally to this work and share first authorship. 
We hypothesized that SWIG could localize the metastasis in the brain parenchyma in real time during surgery and enable identification of the margin and removal of the tumor en bloc, without cutting into it.

\section{Methods \\ Patient Population}

This prospective study was approved by the Fujita Health University Clinical Research Ethics Committee and the Saiseikai Yokohamashi Tobu Hospital. We obtained informed consent from all included patients. We started recruiting the participants in August 2019.

\section{NIR Contrast Agent}

We injected $5 \mathrm{mg} / \mathrm{kg}$ intravenous ICG $\left(\mathrm{C}_{43} \mathrm{H}_{47} \mathrm{~N}_{2} \mathrm{NaO}_{6} \mathrm{~S}_{2}\right.$, Daiichi Sankyo) for 1-2 hours 1 day before surgery. The dose and time were based on previous reports on SWIG. ${ }^{5,6}$ The dose was calculated as $5 \mathrm{mg} / \mathrm{kg}$ per patient, and it was packed into $100-500 \mathrm{ml}$ normal saline solution.

\section{NIR Imaging System}

The VisionSense Iridium camera system and exoscope was used in 5 patients, and the KINEVO microscope system (Carl Zeiss AG) was used in the remaining 5 patients. We calculated the intensity of $\mathrm{T} 1 \mathrm{Gd}$ enhancement by drawing a region of interest (ROI) over the tumor and comparing it with the adjacent brain parenchyma.

The KINEVO microscope comprises a FLOW 800 application that utilizes a filter ranging from $700 \mathrm{~nm}$ to approximately $800 \mathrm{~nm}$. The upper limit corresponded to the excitation wavelength of ICG. The resultant near-infrared (NIR) emission was passed through an 800- to 910-nm filter.

The VisionSense exoscope comprises a silicon image sensor with an open field of view of $19 \times 14 \mathrm{~cm}$ at a 40$\mathrm{cm}$ nominal imaging distance. The emission filter band in the visible light ranged from 400 to $700 \mathrm{~nm}$, whereas that for the NIR was much narrower and ranged from 825 to $850 \mathrm{~nm}$. The camera system featured a dual optical path design, thus allowing separate and independent use of white and NIR light. The presence of separate paths allowed acquisition of faint fluorescent images in the presence of strong white light. A heat map was used as an overlay on the visible-light image to provide quantitative fluorescence intensity.

\section{Data Analysis}

We obtained a background reading from the adjacent normal brain to generate a signal-to-background ratio (SBR). In addition, we used ROI analysis in ImageJ software (NIH) to quantitate the amount of fluorescence from the tissues. Because of the differences between the FLOW 800 in KINEVO and Iridium in VisionSense and the inability to compare the data generated from each system, we used the SBR to reduce errors for each system. We drew 5 ROIs corresponding to the tumor lesions and normal tissue on the images and analyzed their average using Image J software. We conducted univariate analysis using the chi-square or Fisher's exact test for comparing the cat- egorical variables and the unpaired t-test or Mann-Whitney rank-sum test and simple linear regression analysis for the continuous variables. We plotted the unadjusted survival curves by the Kaplan-Meier method, using log-rank tests to assess the significance; $\mathrm{p}<0.05$ was considered significant. We performed the statistical analysis using JMP 14.1.0 (SAS Institute Inc.).

\section{Results}

\section{SWIG Shows NIR Fluorescence of Metastatic Tumor via the Microscope and Exoscope}

There were 4 patients with lung cancer, 3 with colon cancer, 1 with gastric cancer, 1 with urinary tract cancer, and 1 with gall bladder cancer. In the brain, 7 tumors were located in the cranium and 2 in the cerebellum; the remaining lesion spread from the skin to the dura mater. While the average tumor volume was $17,563 \pm 14,669$ $\mathrm{mm}^{3}$, the maximum average tumor diameter was $45.0 \pm$ $14.1 \mathrm{~mm}$. The hematological test results, physical findings, and neurological findings did not reveal any side effects for 3 months after the administration of $5 \mathrm{mg} / \mathrm{kg}$ ICG. In addition, the average time from the administration of ICG to the surgery was $21.1 \pm 1.7$ hours (Table 1 ).

All patients underwent preoperative $\mathrm{Gd}$-enhanced MRI, with the exception of a patient undergoing hemodialysis. NIR fluorescence of the metastatic tumor was identified in all patients, regardless of whether the microscope or exoscope was used during the surgery. The fundamental strategy of tumor resection involved dissecting the outer layers of the tumor, followed by en bloc resection with margins, without invading the tumor cavity. The distances from the brain surface to tumor were $13.0 \pm 4.1 \mathrm{~mm}$ and $8.0 \pm 8.7 \mathrm{~mm}$ in the exoscope and microscope groups, respectively $(\mathrm{p}=0.31)$ (Table 2$)$.

\section{Intraoperative Fluorescence SBR}

The NIR signal was found to be confined to the tumor in all patients. By using SWIG with both the microscope and exoscope, we used the data to determine the presence of NIR fluorescence in the metastatic tumors. The surgeons could visualize the signal following administration of $5 \mathrm{mg} / \mathrm{kg}$ ICG. In all patients, the metastatic tumor produced stronger NIR signals than the surrounding brain parenchyma. While the mean SBR from the brain surface was $1.82 \pm 1.30$, it was $3.35 \pm 1.76$ from the tumor. Hence, the brain surface SBR was $64 \%$ of the tumor SBR (Fig. 1). The depth from the brain surface to the outermost edge of the tumor on Gd-enhanced T1-weighted MRI was 10.56 $\pm 6.74 \mathrm{~mm}$ (range $0-20 \mathrm{~mm}$ ). The deepest tumor $(20 \mathrm{~mm}$ deep) could be also observed from the brain surface. Figure 2 shows the linear regression plot of the SBR of NIR tumor signal versus the depth from the brain surface $(\mathrm{p}=$ $0.031, \mathrm{R}^{2}=0.46$ ). Figure 3 shows the SBR of NIR tumor signal versus the maximum tumor diameter on preoperative T1-weighted MRI $\left(p=0.030, \mathrm{R}^{2}=0.46\right)$. The time (in hours) from ICG infusion to visualization did not reach statistical significance. However, the linear regression plot of the SBR from the NIR tumor signal versus the time from the ICG infusion did not show any significant difference ( $p$ $\left.=0.21, R^{2}=0.22\right)$. NIR spectroscopy (NIRS) did not reveal 
TABLE 1. Data of 10 patients with brain metastasis

\begin{tabular}{|c|c|c|c|c|c|c|c|c|c|}
\hline $\begin{array}{l}\text { Case } \\
\text { No. }\end{array}$ & $\begin{array}{l}\text { Age (yrs), } \\
\text { Sex }\end{array}$ & Location & Pathology & NIRS & $\begin{array}{c}\text { Time From ICG Injection } \\
\text { to Visualization (hrs) }\end{array}$ & $\begin{array}{l}\text { ICG Injection } \\
\text { Dose (mg) }\end{array}$ & $\begin{array}{l}\text { Side } \\
\text { Effects }\end{array}$ & $\begin{array}{c}\text { Max Tumor } \\
\text { Diameter }(\mathrm{mm})\end{array}$ & $\begin{array}{c}\text { Body } \\
\text { Weight }(\mathrm{kg})\end{array}$ \\
\hline 1 & $74, \mathrm{~F}$ & Frontal & Colon & KINEVO & 22 & 250 & No & 40 & 59 \\
\hline 2 & $54, \mathrm{M}$ & Cerebellar & Gastric & KINEVO & 20 & 250 & No & 25 & 72 \\
\hline 3 & $71, \mathrm{~F}$ & Frontal & Colon & KINEVO & 21 & 250 & No & 80 & 50 \\
\hline 4 & $67, \mathrm{M}$ & Occipital & Lung & KINEVO & 20 & 250 & No & 40 & 50 \\
\hline 5 & $80, \mathrm{M}$ & Frontoparietal & Colon & KINEVO & 20 & 250 & No & 50 & 60 \\
\hline 6 & $77, \mathrm{~F}$ & Frontoparietal & Lung & VisionSense & 24 & 200 & No & 40 & 40 \\
\hline 7 & $68, M$ & Frontal & Lung & VisionSense & 20 & 250 & No & 40 & 60 \\
\hline 8 & $81, \mathrm{~F}$ & Frontal dura & Urinary tract & VisionSense & 20 & 200 & No & 45 & 40 \\
\hline 9 & $53, \mathrm{~F}$ & Temporal & Lung & VisionSense & 21 & 250 & No & 40 & 50 \\
\hline 10 & $75, \mathrm{M}$ & Cerebellar & Gall bladder & VisionSense & 22 & 250 & No & 50 & 50 \\
\hline
\end{tabular}

Max $=$ maximum.

any residual tumor after resection. Moreover, postoperative MRI did not detect any residual enhanced lesion.

\section{Ratio of Gd-Enhanced T1 Tumor Signal to Normal Brain on MRI}

It has been reported that preoperative $\mathrm{Gd}$ enhancement may predict NIR fluorescence. ${ }^{2}$ Here, we evaluate the relationships between preoperative MRI findings and the NIR signal. All patients in this study underwent Gd-enhanced MRI, except for 1 patient, who was receiving hemodialysis (case 4). Preoperative MRI comprised the average of the 5 ROIs from the enhanced lesion and normal tissues. The mean ratio of Gd-enhanced T1 tumor signal to normal brain (T1BR) on MRI was $2.43 \pm 1.77$. We conducted linear regression analysis to evaluate the relationships between Gd enhancement on T1-weighted MR images (T1BR) and SBR. Figure 4 shows the linear regression plot of SBR of the NIR tumor signal versus T1BR in 10 patients $\left(p=0.0020, R^{2}=0.77\right)$. In addition, we conducted linear regression analysis to evaluate the relationships between T1BR and the maximum diameter of the tumor.
Figure 5 shows the linear regression plot of the maximum tumor diameter versus T1BR in the aforementioned $10 \mathrm{pa}-$ tients $\left(\mathrm{p}=0.0040, \mathrm{R}^{2}=0.48\right)$.

\section{NIR Differences Between the KINEVO Microscope and VisionSense Exoscope}

Finally, we wanted to check the differences between the KINEVO and VisionSense systems. NIR fluorescence of the tumor could be observed from the brain surface in all patients. While the mean SBR from the brain surface was 1.58 (95\% CI 1.2-2.0) with the KINEVO microscope, it was 2.4 (95\% CI 0.1-5.0) with the VisionSense exoscope. In contrast, those from the tumor itself were 3.50 (95\% CI 0.43-6.57) and 3.23 (95\% CI 0.98-5.48) with the KINEVO and VisionSense systems, respectively. There was no substantial difference between the mean distances from the brain surface to the camera with the microscope (290 mm [95\% CI 188-392 mm]) and exoscope (320 mm [95\% CI 158-481 mm]) ( $\mathrm{p}=0.37)$. Furthermore, the difference between the SBR from the brain surface $(p=0.62)$ and that from the tumor itself $(p=0.90)$ was insignificant.

TABLE 2. NIR signal and MRI Gd-enhanced T1 signal of tumor

\begin{tabular}{|c|c|c|c|c|c|c|c|c|c|c|}
\hline $\begin{array}{c}\text { Case } \\
\text { No. }\end{array}$ & Fluorescence & $\begin{array}{l}\text { NIR } \\
\text { Tumor } \\
\text { SBR }\end{array}$ & $\begin{array}{l}\text { NIR Tumor } \\
\text { SBR From } \\
\text { Surface }\end{array}$ & T1BR & $\begin{array}{c}\text { Gd } \\
\text { Enhancement } \\
\text { on MRI }\end{array}$ & $\begin{array}{c}\text { Signal } \\
\text { Visible w/ } \\
\text { NIR Camera }\end{array}$ & $\begin{array}{c}\text { Distance } \\
\text { From Brain } \\
\text { Surface (mm) }\end{array}$ & $\begin{array}{c}\text { Extent } \\
\text { of Tumor } \\
\text { Resection }\end{array}$ & $\begin{array}{l}\text { Distance Btwn } \\
\text { Camera \& Brain } \\
\text { Surface }(\mathrm{mm})\end{array}$ & $\begin{array}{l}\text { Postop } \\
\text { Therapy }\end{array}$ \\
\hline 1 & Positive & 1.79 & 1.35 & 2.3 & Yes & Yes & 17 & GTR & 300 & RT \\
\hline 2 & Positive & 2.71 & 1.51 & 1.18 & Yes & Yes & 18 & STR & 300 & No \\
\hline 3 & Positive & 6.25 & 1.97 & 1.25 & Yes & Yes & 10 & GTR & 400 & No \\
\hline 4 & Positive & 3.26 & 1.5 & $N A^{*}$ & Yes & Yes & 10 & GTR & 100 & No \\
\hline 5 & Positive & 3.9 & 1.7 & 1.59 & Yes & Yes & 10 & GTR & 400 & No \\
\hline 6 & Positive & 6.32 & 5.02 & 1.27 & Yes & Yes & 0 & GTR & 400 & No \\
\hline 7 & Positive & 2.22 & 1.09 & 1.99 & Yes & Yes & 0 & GTR & 300 & WBRT \\
\hline 8 & Positive & 3.11 & 1.39 & 1.54 & Yes & Yes & 10 & GTR & 350 & No \\
\hline 9 & Positive & 1.7 & 1 & 1.47 & Yes & Yes & 20 & GTR & 150 & WBRT \\
\hline 10 & Positive & 2.81 & 1.54 & 1.22 & Yes & Yes & 10 & GTR & 350 & No \\
\hline
\end{tabular}

GTR = gross-total resection; NA = not applicable; RT = radiotherapy; STR = subtotal resection; $W B R T=$ whole-brain RT .

* This patient underwent hemodialysis and thus Gd could not be used. 


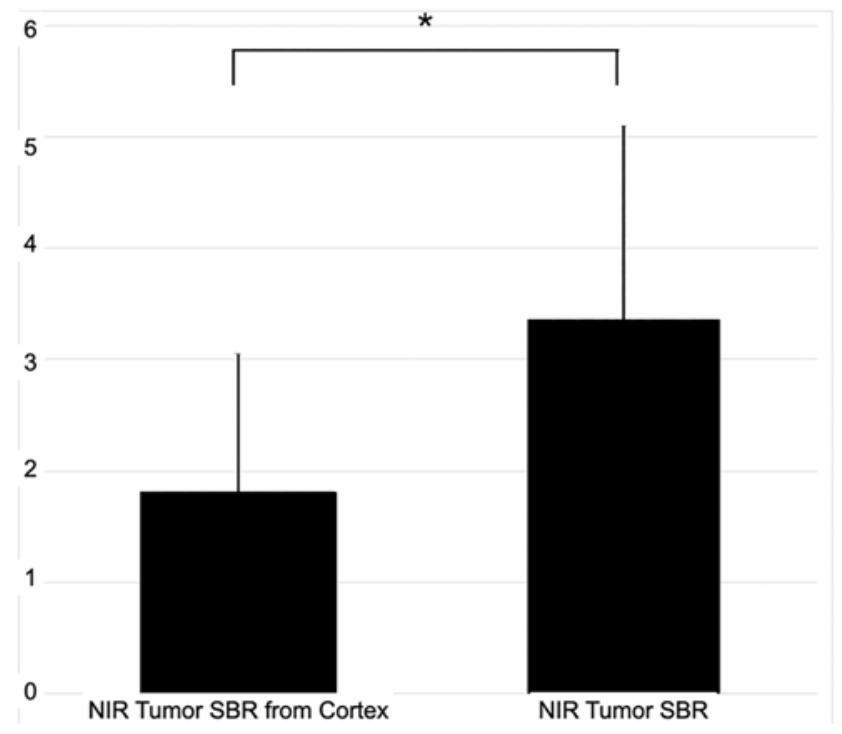

FIG. 1. NIR SBR at different depths at the cortex and at the tumor. The NIR signal is smaller above the cortex; however, it is still visible. Bars represent the means, and error bars represent the SDs. There is a statistically significant difference between the two groups $(p=0.032)$.

Moreover, the distance from the brain surface to the camera system between KINEVO and VisionSense did not show any significant difference $(\mathrm{p}=0.39)$.

\section{Representative Cases \\ Case 1}

A 74-year-old woman presented to our clinic with forgetfulness. She received a diagnosis of brain metastasis from colon cancer. The colon cancer had already been treated surgically and radiologically. The patient was administered $5 \mathrm{mg} / \mathrm{kg}$ ICG for 2 hours 22 hours before the surgery. Preoperative Gd-enhanced MRI showed the ring-

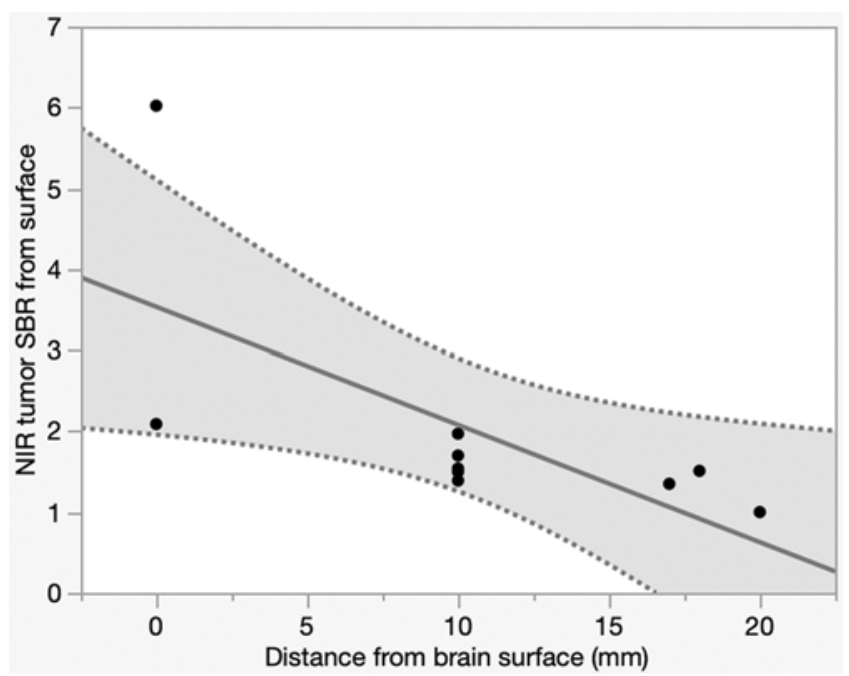

FIG. 2. Linear regression plot of SBR of NIR signal from the tumor versus the distance from the brain surface. The SBR decreases with depth $\left(p=0.031, R^{2}=0.46\right)$.

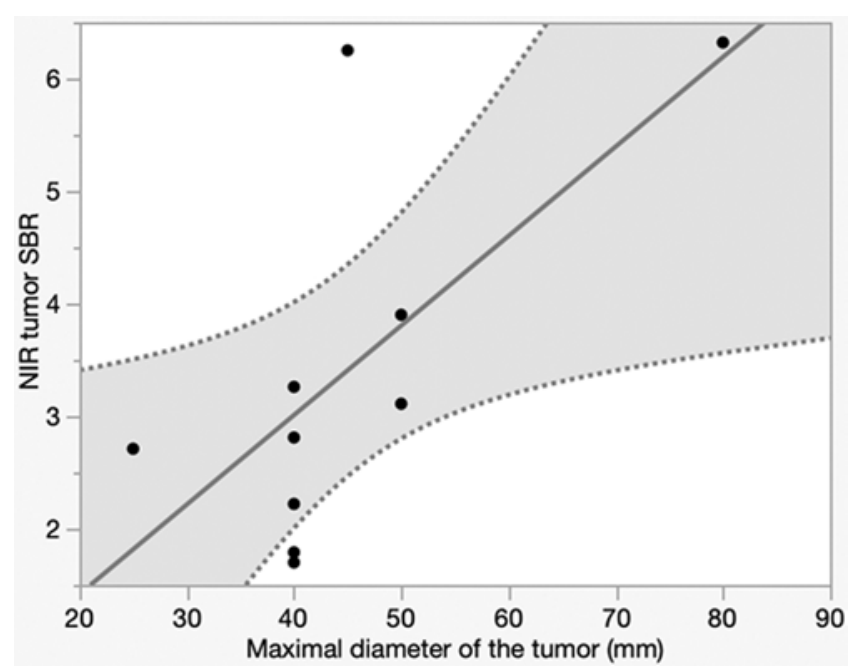

FIG. 3. Linear regression plot of SBR of NIR signal from the tumor versus the maximum diameter of the tumor on preoperative MRI. The SBR increases with the maximum diameter of the tumor $\left(p=0.030, R^{2}\right.$ $=0.46)$.

enhancing lesion with a maximum diameter of $40 \mathrm{~mm}$ in the left frontal lobe (Fig. 6A and B).

The intracranial tumor was operated on using the KINEVO system, and neuronavigation was used to determine the area of the craniotomy and the location of the dural incision. NIR signal could be detected through the brain parenchyma and was reconfirmed by NIRS before the brain incision. The tumor was located at a depth of $17 \mathrm{~mm}$ from the surface and was identified with NIRS (Fig. 6C and D). This was followed by identification of the signal at the tumor (Fig. 6E and F). Following tumor resection with margins, there was no residual tumor and no NIR signal at the end of surgery (Fig. 6G and H). Figure $6 \mathrm{I}$ and $\mathrm{J}$ shows the gross-total resection on postoperative Gd-enhanced MRI. The patient received stereotactic ra-

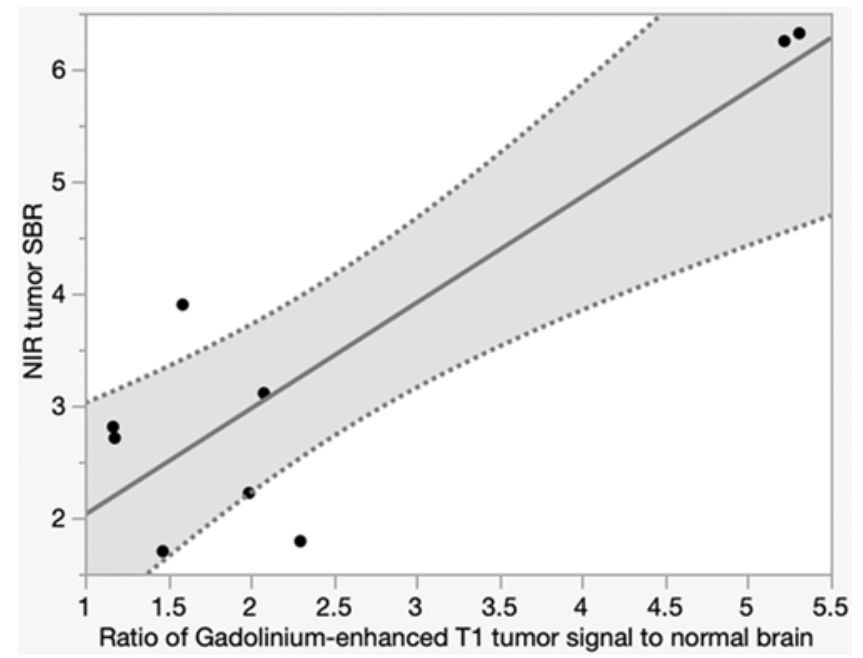

FIG. 4. Linear regression plot of the SBR of the NIR signal from the tumor versus T1BR. The SBR increases with the T1BR $\left(p=0.002, R^{2}=\right.$ $0.77)$. 


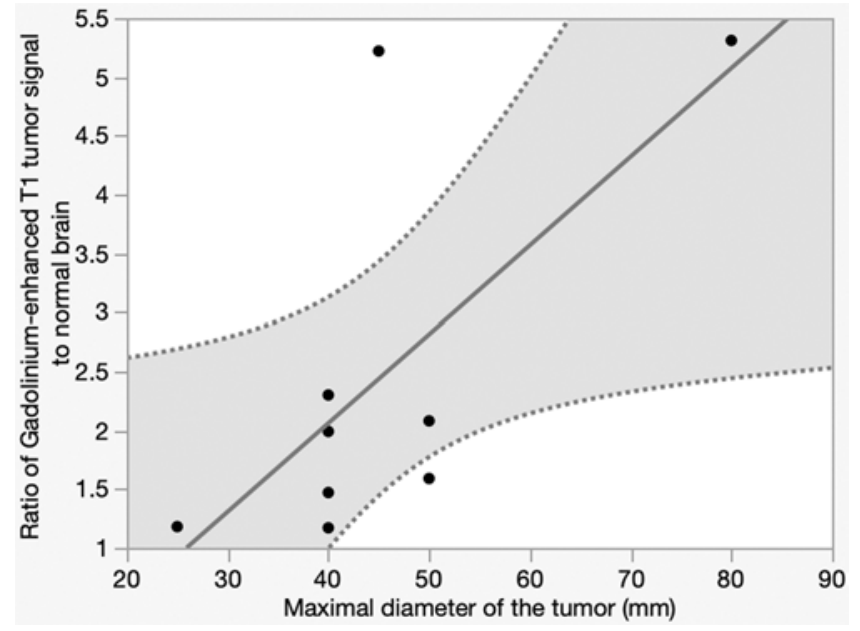

FIG. 5. Linear regression plot of maximum tumor diameter versus the T1BR. The diameter of the tumor increases with the T1BR $\left(p=0.004, R^{2}\right.$ $=0.48)$.

diotherapy following the surgery and there was no recurrence of symptoms for 8 months.

\section{Case 6}

A 77-year-old woman with lung cancer had been treated and followed at a nearby hospital. She presented with severe headache and gait disturbance and was referred to our institution. Gd-enhanced MRI showed an en plaque enhanced lesion with a maximum diameter of $70 \mathrm{~mm}$ in the medial side of the left frontal lobe along with falx and meningeal enhancement, suggesting brain metastasis and meningeal carcinomatosis (Fig. 7A-C). Her intracranial tumor was operated on using the VisionSense system with neuronavigation and NIRS in the same manner as in case 1. The tumor was deeply located along with the falx and was covered by the brain parenchyma (Fig. 7D). It could not be directly observed by the exoscope; however, NIRS could detect the tumor at the cortex (Fig. 7D-F). This tumor identification by NIR continued during surgery and clearly showed residual tumor at the tumor site (Fig. 7G-I). After tumor removal, no residual tumor was observed in either white bright light or NIR (Fig. 7J-L). Postoperative Gd-enhanced MRI showed no enhancing mass except for meningeal carcinomatosis (Fig. 7M-O). The patient's symptoms dramatically improved after surgery, and she was referred back to the nearby hospital for adjuvant radiotherapy.

\section{Discussion}

\section{Benefit of SWIG for the Resection of the Metastatic Tumor}

Neuronavigation is now being used for the removal of intracranial tumors. It facilitates surgical planning before the operation; however, the brain becomes displaced after the dura mater is opened. This in turn prevents determining the accuracy of the tumor location. Thus, the use of an NIR fluorescence technique as an adjunct for tumor removal is beneficial. Previously, researchers have reported on the use of fluorescein and 5-aminolevulinic acid
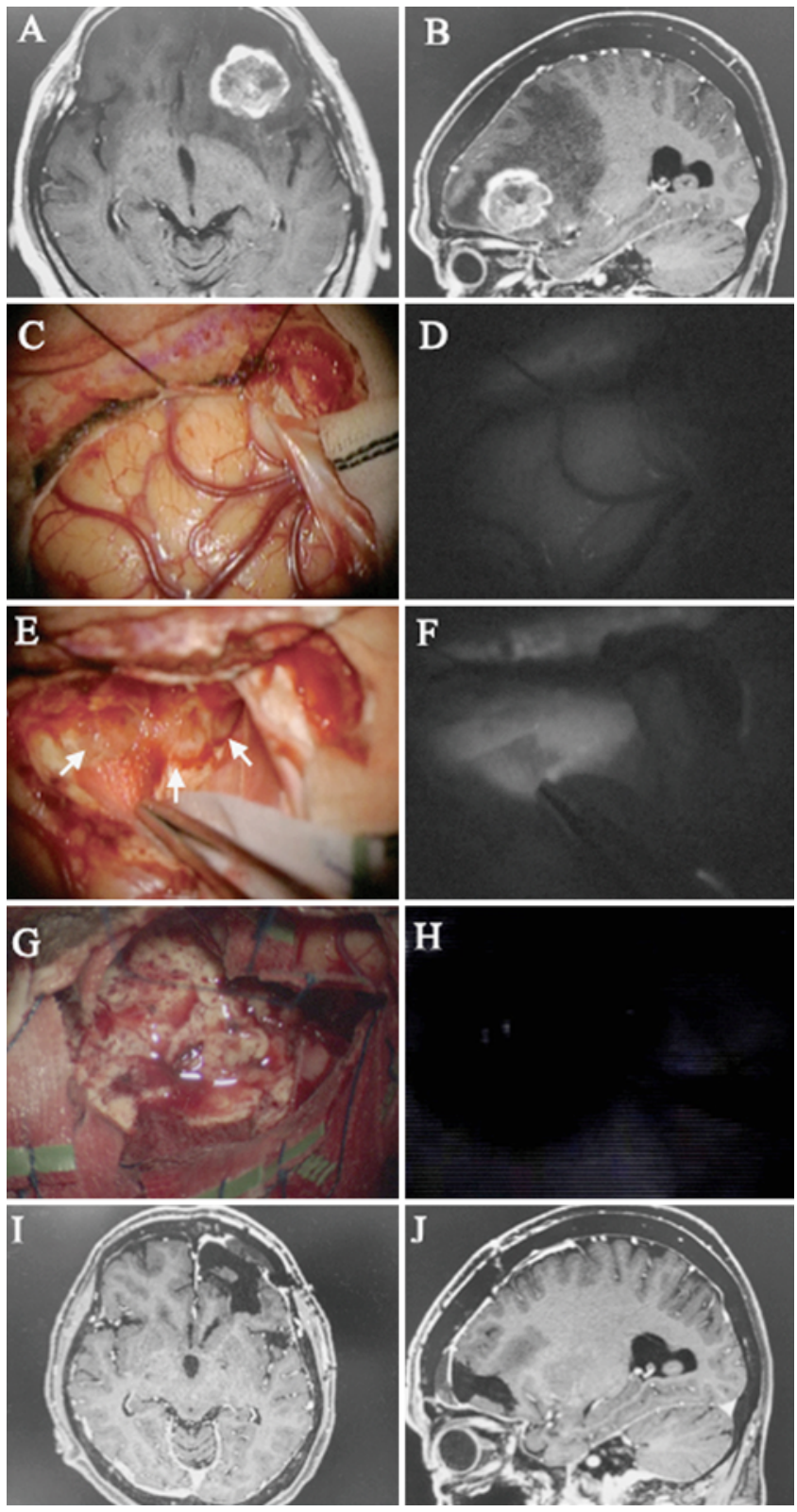

FIG. 6. Case 1. Metastatic tumor from the colon in the left frontal lobe displaying the NIR fluorescence by the KINEVO microscope. A and B: Preoperative axial (A) and sagittal (B) Gd-enhanced T1-weighted MR images showing the ring-enhanced lesion in the left frontal lobe. C: Bright light image on the brain surface. D: NIR fluorescence at the cortex on the brain surface showing the NIR signal through the brain parenchyma, localized to the tumor. E: Bright light image at the tumor. The white arrows indicate the metastatic tumor. F: NIR fluorescence at the tumor showing the NIR signal at the tumor-brain interface. $\mathbf{G}$ and $\mathrm{H}$ : Bright light image $(\mathrm{G})$ and NIR fluorescence $(\mathrm{H})$ at the end of surgery, showing no NIR signal. I and J: Postoperative axial (I) and sagittal (J) Gd-enhanced T1-weighted MR images showing no residual lesion.

(5-ALA) for glioma. However, SWIG with ICG has been used in recent years.

This pilot study confirmed the usefulness of SWIG and ICG in metastatic brain tumors by using an exoscope as well as a microscope. Fluorescein, 5-ALA, and ICG are 

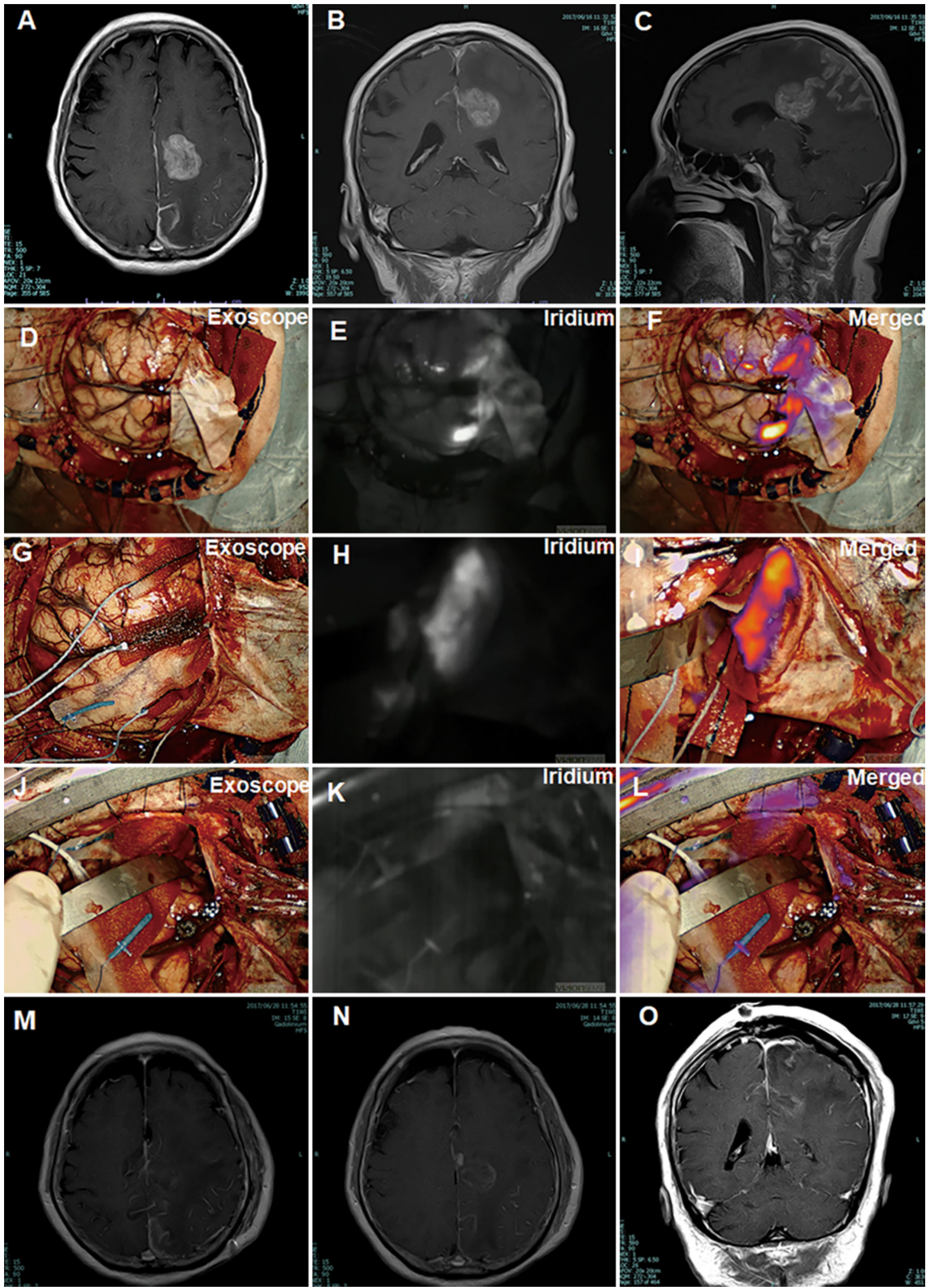

FIG. 7. Case 6. A 77-year-old female with a left frontal metastasis from lung cancer whose surgery was performed with the aid of the VisionSense exoscope. FIG. 7. (continued) $\rightarrow$ 
FIG. 7. A-C: Preoperative axial (A), coronal (B), and sagittal (C) Gd-enhanced MR images revealing a Gd-enhanced lesion along with the falx with perifocal edema. D-L: Surgical findings of the bright field $(D, G$, and J), NIR $(E, H$, and K), and merged views $(F, I$, and $L)$. Before microsurgical manipulation, it is difficult to identify the tumor (D). NIR fluorescence of ICG can show the intraparenchymal metastatic tumor at a lower magnification ( $E$ and $F)$. Tumor removal (G-I). NIR fluorescence can clearly show the tumor in the higher magnification during the tumor removal. After tumor removal (J), NIR fluorescence showed no signal (K and $L$ ). M-O: Postoperative axial (M and N) and coronal (O) MR images showing no Gd enhancement.

currently the available fluorescent agents. ICG has been used as a vascular angiography technique in patients with metastatic tumors. ${ }^{7,8}$ Earlier, $25 \mathrm{mg}$ ICG was intravenously administered to visualize the arterial, capillary, and venous flow. Lee et al. reported on the use of the SWIG technique for metastatic tumor treated by a VisionSense exoscope. ${ }^{3}$ SWIG involves administration of $5 \mathrm{mg} / \mathrm{kg}$ ICG 24 hours before surgery, and 5-ALA helps visualize approximately $62 \%$ of the metastatic tumors. ${ }^{9}$

Kamp et al. cautioned that the residual 5-ALA-induced fluorescence after complete macroscopic resection of a metastasis needs to be cautiously interpreted because of the limited specificity for residual tumor tissue detection. ${ }^{9}$ In that study, approximately $61.5 \%$ of 52 patients showed positive fluorescence for 5-ALA after complete resection under the microscope. In contrast, $42.9 \%$ (18 of 52 cases) showed residual fluorescence of 5-ALA in the resected cavity. Of these patients, 33.3\% (6 of 18 cases) had signs of pathologically confirmed positive tumor cells. Thus, the false-positive rate for 5-ALA-induced fluorescence was $66.6 \%$. Nonetheless, the pattern of fluorescence did not correlate with tumor histology. ${ }^{9}$ Fluorescein was used in fluorescence-guided neurosurgery in one study. ${ }^{10}$ Of the 95 patients with metastatic tumors, $95 \%$ showed positive fluorescence using fluorescein. In contrast, $14 \%$ showed residual tumor on postoperative MRI..1 ${ }^{11}$ Therefore, ICGinduced fluorescence seems to enable more feasible tumor identification in metastatic neurosurgery.

\section{Dose of ICG and Intensity of NIR Signal}

We followed the protocol published by Madajewski et al. and Lee et al. ${ }^{2-5}$ Madajewski et al. reported on administration of a high dose of ICG $(7.5 \mathrm{mg} / \mathrm{kg}) 24$ hours prior to surgery to allow ICG accumulation in the areas of neoplasm in a flank tumor model. ${ }^{5}$ This effect was confirmed by the dose studies in the flank tumor model ${ }^{12}$ and in a murine intracranial tumor model. ${ }^{13}$ Thus, we hypothesized that the accumulation of ICG occurs through an enhanced permeability and retention effect that regulates the solid tumor of enhanced vascular permeability due to defective vascular structures and lymphatic drainage system, breakdown of the blood-brain barrier by the tumor, and increased permeability mediators. Using a boosted light beam is likely to enhance the efficacy of a microscope. ${ }^{14}$ Our findings revealed the availability and usefulness of both the microscope and exoscope for NIRS of metastatic tumor.

\section{NIRS of the Tumor From the Brain Surface}

According to previous reports, NIRS can detect intraaxial tumor at a mean of $13.5 \pm 4.0 \mathrm{~mm}$ in cases of glioblastoma and $6.8 \mathrm{~mm}$ deep in cases of metastatic brain tumors from the brain surface. ${ }^{2,3}$ However, we could vi- sualize all tumors from the brain surface in this study. The depth from the brain surface to the outermost edge of the tumor on Gd-enhanced T1-weighted MRI was 10.56 $\pm 6.74 \mathrm{~mm}$ (range $0-20 \mathrm{~mm}$ ). In addition, we could even observe the deepest tumor with a depth of $20 \mathrm{~mm}$ from the brain surface in case 7. ICG NIRS facilitated visualization of the intraparenchymal lesion through the brain surface, compared with 5-ALA and fluorescein, consistent with the findings of Lee et al. ${ }^{3}$ Thus, the fluorescent signal was visible at the brain surface (64\%) and showed a strong SBR $(100 \%)$ in all cases.

However, an NIR signal can be visible under both a microscope and an exoscope. This in turn might improve the boost excitation of NIRS. In addition, a weak NIR signal can even help identify the tumor. While a stronger NIR signal can result in false-positive findings, a weaker signal produces false-negative results.

\section{Microscope and Exoscope}

$\mathrm{Li}$ et al. reported on the failure of the Leica M530 OH6 to detect ICG fluorescence at the cortex and tumor, with SBRs of $1.8 \pm 0.18$ and $1.7 \pm 0.24$, respectively. ${ }^{14}$ The NIR FL800 OH6 (Leica) has a 300- to 400-W xenon lamp; however, the power of the laser was not known for the KINEVO system. This system was able to detect NIR fluorescence from the brain surface with an SBR of $1.97 \pm 0.3$. In contrast, the SBR from the tumor increased to a mean of $3.43 \pm 0.65$. Thus, the results obtained using the KINEVO system were much better than those obtained using the Leica OH6. Li et al. elucidated the postexcitation boost data with SBRs of $2.8 \pm 0.32$ and $2.1 \pm 0.48$ on cortex and tumor, respectively. ${ }^{14}$ The boosted light was brighter than the original one. Future studies should investigate the differences in signals among the NIR systems. Thus, NIR fluorescence of the tumor can produce the SBRs from the brain surface $(p=0.22)$ and the tumor itself $(p=0.29)$ equally well with the KINEVO and VisionSense systems. While the mean SBR from the brain surface was 1.58 (95\% CI 1.2-2.0) using the KINEVO system, it was 2.4 (95\% CI 0.1-5.0) with VisionSense. In contrast, the mean SBRs from the tumor itself were 3.50 (95\% CI 0.43-6.57) and 3.23 (95\% CI 0.98-5.48) with the KINEVO and VisionSense systems, respectively. Both means are nested within the 95\% CIs for the KINEVO and VisionSense systems, and both systems were more likely to produce similar measurements; however, an equivalency test is yet to be done.

Despite the small sample size, with the KINEVO and VisionSense systems, there was no significant difference between the distance from the brain surface to the camera system (KINEVO, $290 \mathrm{~mm}$ [95\% CI 188-392 mm]; and VisionSense, $320 \mathrm{~mm}$ [95\% CI 158-481 mm]; $\mathrm{p}=0.74$ ) and that to the tumor itself $(\mathrm{p}=0.31)$. Both means are nest- 
ed within the $95 \%$ CIs for the KINEVO and VisionSense systems, and both systems were likely to produce similar measurements. Thus, the KINEVO microscope can allow NIR fluorescence detection of a metastatic tumor. According to Li et al., on boost excitation, the Leica OH6 helped in tumor visualization by increasing the SBR. ${ }^{14}$ SWIG seems to be widely used in brain tumor surgery if the microscope works well without boost excitation.

In future studies, the most appropriate conditions, including time to detection, NIRS machine type, and analysis of software of the various microscopes and exoscopes, should be examined.

\section{T1BR and SBR}

The T1BR has been associated with the intensity of the NIR signal. ${ }^{4}$ Our data demonstrated a correlation between T1BR and SBR $\left(p=0.0020, R^{2}=0.77\right)$ and the maximum diameter of the tumor $\left(\mathrm{p}=0.0040, \mathrm{R}^{2}=0.48\right)$. These results suggest that we can identify the useful SWIG before surgery based on the T1BR on MRI. In addition, the mechanism of Gd-enhanced MRI can only be influenced by vascular permeability. ${ }^{15}$ This supported the hypothesis that accumulation of ICG occurs through the enhanced permeability and retention effect in areas of enhanced vascular permeability. ${ }^{16,17}$ Future studies should aim to verify the available pathology for SWIG and elucidate the mechanism of ICG accumulation.

\section{Possibility of False Positives With the NIR System}

The cameras of the KINEVO and VisionSense systems have an automatic exposure feature that could average the pixel intensity to normalize the background and assign it a neutral gray. The camera tries to balance the exposure when set in the automatic exposure mode. Therefore, we can manually fix the gain and illumination. Automatic exposure can cause high false-positive (i.e., NIR positive and pathology negative for tumor) and false-negative (i.e., NIR negative and pathology positive for tumor) findings at the margins. We had set the percentage of gain and illumination when the tumor was exposed to avoid the false-positive identification of the tumor margins. This in turn was identified by the bright light and NIR fluorescence. Previous reports identified $5.9 \%$ of the true-positive and $48.5 \%$ of the false-positive specimens of metastatic tumor. ${ }^{3}$ However, our results showed that the tumor could be completely resected in 9 of 10 cases under a bright light. In addition, NIR fluorescence could not be detected at the end of surgery. Furthermore, we could not find any enhanced lesion on postoperative MRI with Gd enhancement. This in turn demonstrated the absence of residual tumor after failing to identify an NIR signal. Lee et al. also hypothesized that the time from infusion until imaging can affect the falsepositive margin of the tumor. ${ }^{4}$ Nonetheless, there were no substantial differences between the time from infusion to observation and SBR in our study $(\mathrm{p}=0.20)$.

\section{Limitations of SWIG}

We have not yet evaluated the pathology of all of the specimens and its diagnostic correlation with NIRS findings. Also, the sensitivity and specificity could not be cal- culated in this work. Future evaluations are needed to address these deficiencies.

ICG is neither a receptor-bound nor a receptor-specific agent. Therefore, the lack of specificity is inevitable to this method. We can manually fix the gain and illumination at the tumor site to resolve the problems of the camera gain and autoexposure system as we mentioned above. In addition, we can use these settings throughout the surgery. The scores are the relative values, not absolute values. Thus, the distance between the lens and the target was different every time the tumors were observed by NIR fluorescence. Furthermore, the relative scores were different in the autoexposure system. In addition, we could not decide the absolute value during the surgery. This was a pilot study to establish the protocol for the main study. The small sample size was a major limitation. According to previous reports, there may have been a high potential to decrease the falsepositive rate and autoexposure system. ${ }^{3}$

\section{Conclusions}

This pilot study revealed the usefulness of the SWIG technique for operating on tumor metastases with a microscope and with an exoscope. NIRS with ICG can provide a stronger fluorescence of tumor in relation to normal brain parenchyma. It can also help identify the location of the tumor from the brain surface during the tumor resection. Limiting the autoexposure and fixing the gain and illumination manually at the tumor site may reduce the false-positive rate. Further evaluation is needed to solve the problems and limitations for clinical use; however, the SWIG technique of ICG can achieve safe and complete tumor resection in the near future.

\section{Acknowledgments}

This study was funded by research funding from Fujita Health University.

\section{References}

1. Hojo S, Hirano A: Pathology of metastases affecting the central nervous system. In: Takakura K, Sano K, Hojo S, Hirano A, eds. Metastatic Tumors of the Central Nervous System. Igaku-Shoin; 1982:5-7.

2. Lee JYK, Thawani JP, Pierce J, et al. Intraoperative nearinfrared optical imaging can localize gadolinium-enhancing gliomas during surgery. Neurosurgery. 2016;79(6):856-871.

3. Lee JYK, Pierce JT, Zeh R, et al. Intraoperative near-infrared optical contrast can localize brain metastases. World Neurosurg. 2017;106:120-130.

4. Lee JYK, Pierce JT, Thawani JP, et al. Near-infrared fluorescent image-guided surgery for intracranial meningioma. $J$ Neurosurg. 2018;128(2):380-390.

5. Madajewski B, Judy BF, Mouchli A, et al. Intraoperative near-infrared imaging of surgical wounds after tumor resections can detect residual disease. Clin Cancer Res. 2012; 18(20):5741-5751.

6. Singhal S, Nie S, Wang MD. Nanotechnology applications in surgical oncology. Annu Rev Med. 2010;61:359-373.

7. Ferroli P, Acerbi F, Albanese E, et al. Application of intraoperative indocyanine green angiography for CNS tumors: results on the first 100 cases. Acta Neurochir Suppl. 2011;109: 251-257.

8. Kim EH, Cho JM, Chang JH, et al. Application of 
intraoperative indocyanine green videoangiography to brain tumor surgery. Acta Neurochir (Wien). 2011;153(7):14871495.

9. Kamp MA, Grosser P, Felsberg J, et al. 5-aminolevulinic acid (5-ALA)-induced fluorescence in intracerebral metastases: a retrospective study. Acta Neurochir (Wien). 2012;154(2): 223-228.

10. Okuda T, Kataoka K, Yabuuchi T, et al. Fluorescence-guided surgery of metastatic brain tumors using fluorescein sodium. J Clin Neurosci. 2010;17(1):118-121.

11. Höhne J, Hohenberger C, Proescholdt M, et al. Fluorescein sodium-guided resection of cerebral metastases - an update. Acta Neurochir (Wien). 2017;159(2):363-367.

12. Jiang JX, Keating JJ, Jesus EM, et al. Optimization of the enhanced permeability and retention effect for near-infrared imaging of solid tumors with indocyanine green. Am J Nucl Med Mol Imaging. 2015;5(4):390-400.

13. Zeh R, Sheikh S, Xia L, et al. The second window ICG technique demonstrates a broad plateau period for near infrared fluorescence tumor contrast in glioblastoma. PLoS One. 2017; 12(7):e0182034.

14. Li C, Buch L, Cho S, Lee JYK. Near-infrared intraoperative molecular imaging with conventional neurosurgical microscope can be improved with narrow band "boost" excitation. Acta Neurochir (Wien). 2019;161(11):2311-2318.

15. Montagne A, Toga AW, Zlokovic BV. Blood-brain barrier permeability and gadolinium: benefits and potential pitfalls in research. JAMA Neurol. 2016;73(1):13-14.
16. Maeda $\mathrm{H}, \mathrm{Wu}$ J, Sawa T, et al. Tumor vascular permeability and the EPR effect in macromolecular therapeutics: a review. J Control Release. 2000;65(1-2):271-284.

17. Ergin A, Wang M, Zhang JY, et al. The feasibility of realtime in vivo optical detection of blood-brain barrier disruption with indocyanine green. J Neurooncol. 2012;106(3): $551-560$

\section{Disclosures}

Dr. Lee owns stock options in VisionSense.

\section{Author Contributions}

Conception and design: Muto, Mine. Acquisition of data: Muto, Joko. Analysis and interpretation of data: Muto, Nakagawa. Drafting the article: Muto, Mine, Nakagawa, Joko, Kagami, Inaba, Hasegawa. Critically revising the article: Muto, Mine, Kagami, Lee. Reviewed submitted version of manuscript: Muto, Lee. Approved the final version of the manuscript on behalf of all authors: Muto. Statistical analysis: Muto. Administrative/techni$\mathrm{cal} /$ material support: Muto, Hirose. Study supervision: Hasegawa, Hirose.

\section{Correspondence}

Jun Muto: Fujita Health University, Toyoake-city, Aichi, Japan. muto@fujita-hu.ac.jp. 